\title{
PERCHÉ LEGGERE MICHELSTAEDTER?
}

\section{Giuseppe Stellardi}

\begin{abstract}
La persuasione e la rettorica was written by Carlo Michelstaedter in 1910 as a doctoral thesis, and not intended for public consumption. It has, however, acquired the status of a significant philosophical text of the early $20^{\text {th }}$ century. The present article attempts to elucidate the reasons why the book, almost one hundred years later, and in spite of its apparently outdated style, content and context, is still relevant. It does so through an investigation concerning its intended readership, and an analysis of its two main wavelengths: the sociological one (criticism of contemporary pedagogy and social structures) and the philosophical one (pursuit of the "thought of the limit").
\end{abstract}

"Egli deve aver il coraggio di sentirsi ancora solo, di guardar ancora in faccia il proprio dolore, di sopportarne tutto il peso. (Michelstaedter, 1982: 83)

Chi ne abbia tentato la lettura sa bene che il piccolo opus magnum di Carlo Michelstaedter è un osso duro per chiunque. Ma se $L a$ persuasione e la rettorica ${ }^{1}$ non è un libro facile, non è solo per ragioni di forma (lingua e stile) e contenuto (idee). Che altro c'è in un libro, ci si chiederà, oltre a forma e contenuto? C'è un lettore, o forse anche più

Cito dall'edizione Adelphi del 1982, curata da Sergio Campailla (d'ora in poi abbreviando in Michelstaedter, 1982). Nelle citazioni, il corsivo è sempre dell'autore. 
di uno: il lettore immaginario che ogni libro (talvolta all'insaputa dell' autore) configura e invoca, e quello reale (non sempre lo stesso) che ogni libro trova, o perde ${ }^{2}$. Fra le molte difficoltà presentate dall'opera di Michelstaedter questa non è la più piccola: non è sempre chiaro a chi sia veramente indirizzata, né chi (e per quali motivi) essa possa riuscire a attrarre nella sua orbita; specialmente oggi, a quasi un secolo dalla composizione, e in tanto diverso contesto storico e culturale.

Cominciamo dunque col ricordare brevemente le circostanze in cui il libro fu concepito e realizzato ${ }^{3}$. Come è noto, La persuasione e la rettorica è una tesi di laurea; fra i molti interessi coltivati da Michelstaedter (disegno, poesia, matematica, musica, nuoto), la filosofia si cristallizza negli anni del soggiorno fiorentino (1905-1910) come il campo prescelto ${ }^{4}$ in cui il giovane e sconosciuto studente goriziano fornirà la prima prova "ufficiale" del suo già indubbio talento. Alla fine dell'estate del 1910 Michelstaedter compie il passo finale e porta a termine la dissertazione; ma ha appena completato il lavoro (con ampie appendici e tesine aggiuntive, come da regolamento) quando si suicida in casa sua, a Gorizia, il 17 ottobre. Ha solo ventitre

2 In tempi recenti, spetta a Umberto Eco, in particolare, il merito di aver sinteticamente presentato a livello teorico le nozioni di "lettore implicito", "lettore modello" e "lettore empirico". Si veda in proposito, per esempio, il suo I limiti dell'interpretazione (Eco, 1985).

3 Per motivi di spazio e opportunità, non tento neppure di disegnare in questa sede il profilo dettagliato dell'opera, né del contesto locale e europeo da cui emerge. Rimando invece alla recente bibliografia critica in proposito, e specialmente: Campailla, 1973 e 1981, Brianese, 1985, Semeraro, 1986, Cerruti, 1987, Muzzioli, 1987, Pieri, 1989, Bini, 1992, Arbo, 1996, Negri, 1996, Michelis, 1997, Raschini, 2000, Taviani, 2002, Stella, 2002. Un contributo particolarmente innovativo e decisamente comparativo alla contestualizzazione dell'opera del goriziano è stato fornito da Harrison, 1996.

4 Ma si può anche supporre che, alla radice della "sindrome di Michelstaedter", ci sia proprio l'impossibilità fondamentale di scegliere fra le infinite strade proposte dalla vita: "E come se mille prospettive lo invitassero a dover compiere delle scelte che lui no può, o che non sa fare" (Innella, 1995: 45). 
anni, la stessa età a cui si era tolto la vita, quasi esattamente sette anni prima, Otto Weininger.

L'opera (che non era dall'autore destinata alla pubblicazione) fu stampata per le cure degli amici e attrasse subito l'attenzione di alcuni eminenti contemporanei ${ }^{5}$, poi, nonostante brevi riapparizioni nella forma di alcune ristampe e riedizioni, cadde per lunghi periodi nel dimenticatoio; in tempi più recenti, grazie alla raccolta in un volume di un'ampia scelta degli scritti a cura di Gaetano Chiavacci (Michelstaedter, 1958) ${ }^{6}$ e, soprattutto, all'instancabile attività editoriale e critica di Sergio Campailla, l'interesse per il goriziano, e principalmente per la Persuasione, ha vissuto una stagione di rinascita. Oggi Michelstaedter è una presenza discreta ma non più marginale nel contesto della letteratura e del pensiero italiani e europei ${ }^{7}$.

5 Fra cui Giovanni Papini, che del resto a Michelstaedter aveva già dedicato un articolo subito dopo il suicidio, e ancor prima di aver potuto leggere l'opera (Papini, 1910); e poi Borgese, Gentile, Prezzolini, Tilgher.

6 Soprattutto notevoli, oltre all'opera principale, il Dialogo della salute e le poesie sparse.

7 Esistono da tempo traduzioni della Persuasione in tedesco, francese e spagnolo; quanto al mondo anglosassone, una traduzione americana è venuta recentemente a colmare una vistosa lacuna (Michelstaedter, 2004), mentre un'altra versione in inglese dovrebbe essere disponibile prossimamente. 
Un primo elemento può dunque essere acquisito agli atti: il lavoro aveva una destinazione, e dunque, presumibilmente, un "lettore implicito", ben precisi: sarebbe stato letto in prima istanza, e valutato, da una commissione di docenti universitari, specialisti nel campo degli studi filosofici. ${ }^{8}$ Nella maggior parte dei casi, questo basterebbe ampiamente a fornire al lettore moderno il profilo generico e dunque la chiave di lettura di un'opera: le tesi di laurea in filosofia hanno di solito una forma, un tenore, un sapore specifici. Rispettano abitudini formali consolidate dall'uso e regole di comunicazione, argomentazione e presentazione per lo più implicite, ma abbastanza ferree $^{9}$.

Ora, balza subito agli occhi che La persuasione e la rettorica, almeno per un lettore dei giorni nostri, non è una normale tesi di laurea. A questo punto, naturalmente, bisognerà tener conto della distanza cronologica e di un eventuale sfasamento culturale: non è improbabile che nell'anno 1910 le circostanze attinenti alla composizione di una dissertazione accademica fossero un po' diverse da quelle attuali. Il contesto istituzionale, sociologico e ideologico del lavoro intellettuale in ambito universitario avrà avuto poco in comune con quello - ipercodificato, cristallizzato e quindi maggiormente omogeneizzato - degli atenei di massa dei giorni nostri; il rapporto coi docenti sarà stato senz'altro meno impersonale; e all'atto della scrittura, lo studente avrà probabilmente avuto maggiore libertà, a livello tanto di contenuto quanto di forma. Forse quindi una

8 Michelstaedter, tuttavia, al momento di depositare la tesi, dimostra una singolare (e caratteristica) noncuranza per gli aspetti più pratici e burocratici della faccenda: "lo non mi sono inteso con nessun professore quanto a questo lavoro, né ora saprei dargli alcun indirizzo. Faccia dunque Lei come meglio crede. Quello che mi sta a cuore è d'esser sbrigato prima della fine del mese corrente" (lettera del 5 ottobre 1910 al Segretario dell'Istituto di Studi Superiori, in Michelstaedter, 1958: 622).

9 Ancora una volta, si da il caso che sia Umberto Eco ad aver codificato esplicitamente alcune di queste norme; si veda Come si fa una tesi di laurea (Eco, 1985). 
dissertazione di laurea sarà stata, più spesso di quanto non avvenga oggi, una vera tesi, con un tenore di originalità, eccentricità e aggressività assai superiore a quello raccomandabile ai giorni nostri. ${ }^{10}$

Anche tenendo in debito conto tutto ciò, resta tuttavia difficile identificare il lettore della Persuasione semplicemente con il professore universitario in funzione di esaminatore. Perché? Cerchiamo di evidenziare le caratteristiche dell'opera che visibilmente esorbitano dalla sua configurazione primaria.

Già a partire dal titolo, se non mi inganno, si percepisce un tono asseverativo e dialettico, più che scolastico o erudito; si sente il premere di una questione fondamentale; si intuisce che il campo d'indagine sarà ampio, e che l'autore si occuperà più di un vero problema, che delle minuzie filologiche o concettuali entro le quali spesso i problemi si esauriscono. Il tema stesso, nella sua eroica semplicità, suggerisce coinvolgimento personale e intensità di sentimenti, invece che "distacco professionale" e oculata parcellizzazione dello sforzo intellettuale. C"è un che di radicale e militante, in questo titolo, e il sapore di una sfida, non puramente accademica. Il lettore intelligente non mancherà di sentirsi oscuramente messo in causa e di prepararsi a una lettura che fin dall'inizio si annuncia come poco riposante.

10 Che avranno pensato di quella tesi i professori del tempo? In ogni caso, sospetto che oggi il lavoro avrebbe poche speranze di superare l'esame con esito favorevole, o anzi di raggiungere lo stadio finale nella procedura: il relatore insisterebbe, come minimo, su una presentazione formalmente più standardizzata, e emotivamente meno coinvolta. 
Sulla soglia del testo stesso, l'esergo dall'Elettra di Sofocle e la breve "Prefazione" provvedono a mettere definitivamente in allarme, se non in fuga, il lettore che ancora sperasse di avere per le mani una normale, diligente, innocua tesi di laurea. "So che faccio cose inopportune e a me non convenienti", confessa Michelstaedter per bocca di Elettra (Michelstaedter, 1982: 33); e poi, lasciata la maschera trasparente della citazione, attacca subito a sua volta in prima persona, e in modo singolarmente enigmatico e inquietante: "Io lo so che parlo perché parlo ma che non persuaderò nessuno; e questa è disonestà - ma [...] «è pur necessario che se uno ha addentato una perfida sorba la risputi»" (35): e siamo già arrivati al cuore della faccenda, nel territorio eccitante e pericoloso in cui la filosofia e la vita si confondono, coincidono. Abbiamo appena cominciato a leggere e già sappiamo che i pensieri di questo studente non sono cosa da tavolino e da esame, non si restringono alla cerchia sterilizzata e impersonale della ricerca erudita, e anzi coinvolgono direttamente l'esistenza di chi scrive. Qui non è il caso di aspettarsi una giudiziosa separazione fra la sfera della riflessione intellettuale e quella dell'azione pratica. Se il primum vivere, deinde philosophari ${ }^{11}$ ha qui un senso, non è quello comune e filisteo ("la filosofia va bene, però prima di tutto bisogna pensare a pane e companatico"), ma invece quello eroico e impegnato ("solo una filosofia che rifletta la vita, che sia la vita, è degna di essere abbracciata").

Per quanto riguarda il tenore scientifico dell'opera, i riferimenti specialistici certo non mancano, e anzi la tesi trabocca di erudizione, soprattutto per quanto riguarda le fonti classiche, e l'uso della lingua greca in generale; ma, paradossalmente, è proprio la straordinaria familiarità coi greci e col greco antico a suscitare la sensazione precisa

11 Per un'utile discussione dell'antico motto, con particolare riferimento a Hegel, Kierkegaard e Schopenhauer, si veda Giuseppe Tortora, "Kierkegaard and Schopenhauer on Hegelianism", in Metalogicon, VII, 1, 1994: 69-84 (disponibile anche su internet: http://www.filosofia. unina.it/tortora/saggi/kierkegaard.html). 
di un rapporto con la materia trattata che eccede i limiti dell'accademia ${ }^{12}$. Michelstaedter in verità non "cita", ma invece conversa, interpella, apostrofa, tanto in italiano quanto in greco; e sempre chiama in causa, mettendosi in causa allo stesso tempo. E la deprecazione, l'esempio o l'apologo prevalgono regolarmente sul sillogismo e sulla dimostrazione.

Manca evidentemente un metodo chiaro, una struttura solida, e un'argomentazione logica; benché, in superficie, sia suddivisa in parti e capitoli ben definiti, la tesi procede arbitrariamente, non costruisce un edificio discorsivo equilibrato, ma invece naviga inesorabilmente, si direbbe, fra scoglio e scoglio, verso la propria estenuazione. In effetti, manca una conclusione - se non proprio condivisibile - almeno plausibile. La parte finale, infatti, ripropone (in modo non meno perentorio e assai più concreto) il divario fra pensiero e vita che si era annunciato dall'inizio come il problema di fondo, e che - ora scopriamo - è rimasto tale, irrisolto, fino alla fine dell'opera; dove Michelstaedter, con sarcastico candore, mette in scena, sotto il velo leggerissimo della terza persona, la propria condizione di studente alle soglie della vita e della carriera:

"«Altro è la teoria, altro la pratica».

«... Tu devi fare uno studio su Platone o sul vangelo» gli diranno «è perché così ti fai un nome, ma guardati bene dall' agire secondo il vangelo [...]» Così si conforta il giovane a perseguire nel suo studio scientifico senza che si chieda che senso abbia [...].»" (Michelstaedter, 1982: 189)

12 È tipica di Michelstaedter una appassionata consuetudine con la tradizione filosofica classica, che però ostinatamente rifugge dal seguire i binari mentali e le abitudini dell'accademia: "Sin dagli anni liceali Michelstaedter aveva mostrato straordinarie doti per lo studio del greco, della matematica, della filosofia, ma aveva sempre rifiutato lo studio erudito, la puntigliosità filologica fine a se stessa, la ricerca archivistica" (Toni lermano, in AA.VV., 1994: 9). 
Sta parlando ai suoi professori, questo giovane e impertinente studente? In ogni caso, qui la tesi, tornando al suo punto di partenza (la vita medesima del proprio autore, i suoi personali dilemmi, il nodo tragico dell'apparire e dell'essere) si richiude inevitabilmente su se stessa. Il lavoro, in effetti, non termina con un'affermazione conclusiva, ma invece, assai bruscamente e inaspettatamente, finisce ripiegandosi sull'atteggiamento pessimistico e quietamente disperato dell'inizio:

"Ma gli uomini temo che siano sì bene incamminati, che non verrà loro mai il capriccio di uscir della tranquilla e serena minore età." (ibidem)

Sono le ultime parole di Michelstaedter. Qualunque fosse il messaggio, e chiunque fosse il destinatario immediato di questo sforzo estremo, l'autore non conta molto sulla possibilità di averlo convinto. Michelstaedter ha certamente risputato la sua "perfida sorba", ma non ha veramente dimostrato la sua tesi: ha invece semplicemente esaurito la propria disponibilità a cercare (vanamente) di farlo; ma altro esito non si poteva dare, date le premesse, come vedremo.

Il lettore primario, se si tratta di un'onesto professore universitario, col compito da valutare forme e contenuti secondo criteri prestabiliti, è dunque forse perduto fin dall'inizio: troppo solitario il procedere, e non quello di uno studente "laureabile". Troppo idiosincratici i modi, e inquietanti (anche perché mai dichiarati) i fini: che va cercando questo studentello? Certo molto più di un certificato di laurea....

$\mathrm{Ma}$, se il professore recede dall'orizzonte comunicativo di questo strano libro, un altro lettore è immediatamente configurato ${ }^{13}$. L'altro e più segreto lettore che il libro invoca, tuttavia, è multiplo e difficile da

13 Non parlo di lettori fisici, naturalmente: così, è possibilissimo che il lettore "primario" e il lettore "altro" si incarnino fisicamente nello stesso professore universitario, per esempio. 
definire. Se ne esaminiamo le componenti, possiamo immaginare che si stratifichi su almeno tre livelli chiaramente differenziabili: 1) l'autore stesso, 2) amici e seguaci a lui contemporanei, 3) la posterità.

$\mathrm{Su}$ uno dei molteplici livelli dell'opera, Michelstaedter indubbiamente parla a se stesso. Nonostante l'(ironicamente) ostentata auto-legittimazione ufficiale in quanto tesi di laurea (Michelstaedter, 1982: 36), e lungi dal soddisfare semplicemente un'imposizione estrinseca, la Persuasione manifesta un'intima necessità espressiva e dialettica; il coinvolgimento personale, evidente fin dall'inizio, e la pessimistica sicurezza di non poter convincere nessuno, indicano che il libro è certamente (e forse soprattutto) un dialogo dell'autore con se stesso, un tentativo di auto-chiarificazione e auto-affermazione, verosimilmente anche uno sforzo teso a risolvere i paradossi e vicoli ciechi del proprio pensiero. Michelstaedter, sapendo di non poter essere seguito sul difficile cammino intrapreso, camminando parla da solo, almeno in parte. Questa disposizione meditativa e quasi solipsistica del testo risulta in certo modo intensificata dalla tragica fine dell'autore: con l'atto stesso del suicidio, infatti, Michelstaedter sigilla la solitudine estrema del proprio cammino e sottrae se stesso (e, per quanto può, l'opera) alla dimensione del commercio ordinario con $\mathrm{i}$ propri simili. Se ci saranno lodi o critiche, autore e opera ne saranno perpetuamente indenni. Ciò che è detto, è detto una volta per tutte, senza revisioni, ripensamenti o compromessi possibili, e senza spazio di replica.

Ma nessuna opera può esistere veramente senza un lettore; se autore e testo perdono il primo (cronologicamente) fra i propri lettori impliciti, se dunque l'autore non potrà più leggersi, parlarsi, comprendersi, correggersi, spiegarsi, difendersi, l'opera non può che affidarsi, per essere letta e compresa, dunque per esistere oltre la morte dell'autore, ad altri lettori, gli unici rimasti: i suoi contemporanei, e la posterità. È così che Michelstaedter, uccidendosi, e dunque sottraendosi per sempre al dialogo, carica i sopravvissuti, gli orfani, i suoi lettori, della responsabilità morale di non lasciar morire la sua 
voce. E non è questa, in fondo, per quanto amplificata nel caso specifico, la struttura e vocazione di ogni scrittura? Non si scrive forse, sempre, per obbligare qualcuno a leggere ${ }^{14}$ ?

Ma questo lettore altro, se l'opera è destinata a sopravvivere, deve già essere nel testo, da sempre in attesa, e pronto a rispecchiarsi nel lettore empirico che forse un giorno, aperte quelle pagine, vi troverà se stesso. Non è dunque vero, a un più approfondito livello d'analisi, che Michelstaedter parli da solo; né mai potrebbe esserlo, per qualunque fattispecie di scrittura, anche la più introspettiva e autistica. Si scrive sempre per l'altro; o, per lo meno, l'altro non è mai assente all'atto della scrittura.

14 Una situazione simile costituisce il punto di partenza del pirandelliano I giganti della montagna: anche lì, tutto ruota attorno al rapporto fra una sopravvissuta (Ilse) e un autore morto (e anche in quel caso suicida), come a suggerire il nodo indissolubile di amore, morte, piacere e colpa che si trova alla radice di ogni creazione artistica. 
Per quanto concerne i suoi primi lettori, Michelstaedter ha avuto la fortuna di trovarli in un manipolo di amici sinceri, senza i quali probabilmente il suo nome e la sua opera sarebbero morti insieme a lui: Vladimiro Arangio Ruiz, Gaetano Chiavacci, il giovane cugino e discepolo Emilio Michelstaedter raccolsero il lascito bruciante quasi dalle mani stesse dell'autore, e per loro l'opera scritta portò sempre impressa indelebilmente la voce e l'orma di un corpo fisico. Proprio questo, però, se li autorizza in quanto testimoni, inquina di forti valenze emotive il loro rapporto col testo. Influenzati dalla persona, oltre e forse più che dall'opera, di Carlo Michelstaedter, gli amici sono sovrastati dalla personalità eccezionalmente carismatica di quest'ultimo, dalla tragedia del suo gesto finale; tutto questo può inquadrare la lettura, e limitarla, incatenarla al suo contesto di partenza. Il lettore contemporaneo, il lettore amico, per quanto certamente collocabile nell'orizzonte di lettura previsto o sperato, non può essere il destinatario essenziale di un testo come la Persuasione, così come non può esserlo il professore universitario, né lo stesso autore ${ }^{15}$. E in ogni caso, anche quella generazione di lettori privilegiati è a sua volta scomparsa.

Resta quindi un unico lettore perennemente superstite, e sempre in ascolto: il lettore futuro. Siamo (per ora) noi, e sono (per sempre) i posteri. Quelli che presumibilmente a Michelstaedter stava a cuore di convincere ad "essere convinti". Quelli che poteva immaginare capaci, contro ogni speranza, in un diverso e inimmaginabile contesto, di capire. È a un futuro e improbabile lettore che quel cadavere così ingiustificabile chiede di ascoltare la sua voce così poco carezzevole, così disperata, e di tentare, almeno, la lettura di un'opera

15 Anche quella del "destinatario essenziale", o "lettore puro", è, naturalmente, una figura virtuale: il lettore puro, in particolare, non esiste, perché coinciderebbe, paradossalmente, col lettore privo di contesto: quindi, col lettore che non può leggere (o capire) il testo. Il lettore puro è il lettore cieco, sordo e muto: quello che, davanti al testo, non sa di averlo davanti a sé. Quello che, avendo davanti a sé un testo, non sa che farsene. 
apparentemente tanto inattuale. Ma il dovere morale nei confronti di un morto non può da solo giustificare e sostenere la lettura. Bisogna che nel testo ci sia qualcosa di ancora vitale, qualcosa che ci parli non della morte del suo autore, ma della nostra vita. E arriviamo al nocciolo della questione: che cosa dice, Michelstaedter, che ancora abbiamo bisogno di sentirci dire? E chi siamo noi, se la sfida di Michelstaedter ci può ancora coinvolgere? Se quel morto ancora riesce, nel frastuono mediatico e cibernetico, a farsi leggere, a farsi ascoltare da noi?

Anche questo "noi", però, a un più attento esame finisce per sfaldarsi. Il lettore postumo, quello che il libro incontra solo dopo la morte dell'autore, si suddivide ulteriormente: per esempio, c'è il lettore accademico specializzato, per cui La persuasione e la rettorica sarà solo un interessante documento da studiare in relazione ad altri documenti dello stesso genere o della stessa epoca; è questa una forma d'ascolto perfettamente legittima ed anzi indispensabile, naturalmente. C'è poi il lettore "interessato", quello che per caso trova in Michelstaedter conferma di ciò che già sa, e lo usa proprio allo scopo di rafforzare le proprie posizioni ${ }^{16}$; e anche questo, beninteso, è un tipo di lettura legittimo e proficuo. E poi, finalmente, c'è il lettore (non "puro" ma, fra pesanti virgolette) "libero": quello nel cui orecchio, sgombro di doveri e interessi, il messaggio lanciato da Michelstaedter un secolo fa ha la possibilità di risuonare ancora, di generare un fremito d'ascolto genuino.

Siamo arrivati quindi al lettore che questo libro merita: qualcuno che sia disposto a fare lo sforzo di andare oltre i limiti di ciò che pensa, di lasciarsi sfidare a tutti i livelli (sintattico, intellettuale, morale); che non affronti il testo con l'intenzione di inquadrarlo, utilizzarlo, neutralizzarlo; soprattutto, che sia preparato a accettare di non trovare, nel libro, risposte alle domande che il libro stesso suscita, e che nel

16 Penso ad esempio alla lettura apertamente politicizzata in chiave anti-bellica di Michelstaedter. Si veda Fausto Bertinotti e Alfonso Gianni, Per una pace infinita, Ponte alle Grazie (2002). 
lettore trovano una camera di risonanza. Ma quali sono queste domande? Qual è il messaggio che nel lettore deve risuonare? Anche qui bisogna fare delle distinzioni,

Da un lato, la pars destruens (a) della fatica del goriziano è sufficientemente chiara, e facilmente collocabile all'interno di coordinate storico-culturali: Michelstaedter ce l'ha con la morale comune, col sistema educativo, con l'ipocrisia, col conformismo sociale, con il cieco perseguimento degli obbiettivi del cosiddetto progresso scientifico, con l'universale integrazione tecnocratica, con la burocrazia, con la falsa sicurezza che spegne l'intensità della vita. Esprimendo inquietudini che eccedono i limiti della sua persona, si fa portavoce di una corrente (minoritaria ma ben attestata) di critica radicale dell'intero modo di vita delle società occidentali avanzate.

La pars construens (b), d'altra parte, è chiara solo in termini di ideali irrealizzati, forse utopici, forse mostruosi nelle loro conseguenze ultime: essenzialmente, l'ideale di un essere umano che non sia amputato - ad opera della società - del proprio potenziale creativo, l'ideale di una vita in più intimo contatto con la natura e con se stessa, anche a rischio della propria morte; l'ideale di un più autentico rapporto con i propri simili. Di questo ideale ci sono nel libro vari esempi eformulazioni ${ }^{17}$, ma non una definizione compiuta e dettagliata, né la la prova argomentata della sua possibilità pratica a livello sociale (ossia oltre la sfera dell'individuo isolato $)^{18}$. Bisogna chiedersi: è compatibile, questo ideale, con un'organizzazione sociale che non sia

17 Per esempio: "Questo so che la mia coscienza, corporea o animale che sia, è fatta di deficienza; che l'Assoluto non l'ho finché non sono assoluto, che la giustizia non l'ho finché non sono giusto, che la Libertà, il Possesso, la Ragione e il Fine non li ho finché non sono libero e finito in me stesso e non manco di niente, che mi si finga a fine nel futuro, ma ho il fine ragionevole ora qui tutto nel presente, non aspetto, non cerco, non temo, ma sono persuaso" (Michelstaedter, 1982: 96).

Si veda a titolo d'esempio questa drastica affermazione, riferita al persuaso: "[...] egli è solo nel deserto, e deve crear tutto da se: dio e patria e famiglia e l'acqua e il pane" (Michelstaedter, 1982: 70). 
selvaggiamente irrazionale, disastrosamente improvvida, e in fin dei conti destinata all'auto-distruzione?

Quel che resta comunque interamente oscuro è il processo di transizione da $(a)$ a $(b)$, e se tale transizione sia possibile in pratica. Abolite, per ipotesi, le strutture sociali tanto aborrite da Michelstaedter, che direzione prenderebbe la specie? Quella della piena realizzazione del proprio potenziale espressivo, o invece - nonostante le migliori intenzioni dell' autore - quella del ritorno all' animalità più brutale? Ma sarebbe realmente possibile abolire quelle strutture, senza qualche forma di terrificante ingegneria sociale? $\mathrm{E}$, in tal caso, non sarebbe lo sforzo liberatorio vanificato in partenza dalla necessità di applicare pesanti mezzi a fini troppo discosti dalle abitudini sociali acquisite?

Michelstaedter non si premura di offrire prove o almeno argomentazioni che possano costituire una forma di sostegno alle sue tesi. Non si sofferma neppure a considerare se una società basata su simili ideali sia moralmente accettabile, di fatto praticabile, o perfino logicamente possibile. Sembra dunque impossibile poter inquadrare la Persuasione in un'ottica di pensiero sistematico ${ }^{19}, \mathrm{o}-$ tanto meno - di proposta politica; ma allora, qual è la lunghezza d'onda portante di questo libro? Quali corde profonde tocca?

Si dirà che Michelstaedter manifesta il disagio o la malattia di un'intera epoca ${ }^{20}$, e questo è certamente vero, oltre che storicamente interessante, e forse perfino utile alle generazioni future. $\mathrm{Ma}$, in primo luogo, possiamo veramente dire che quella speciale condizione

\footnotetext{
19 "Egli finisce così per apparirci [...] impegnato contemporaneamente in direzioni teoretiche molteplici e talora potenzialmente divergenti, che rendono ardua la sua collocazione tra i vari «ismi» disponibili, e spiegano la sufficienza con cui è stato a volte giudicato dai filosofi più legati all'ideale della sistematicità e del rigore dialettico - ad esempio, da Gentile" (Michelis, 1997: 136).

20 Harrison, per esempio, assume apertamente e senza remore questa prospettiva sintomatologica, descrivendo il proprio approccio come "[...] not a study of Michelstaedter so much as a symptomatology of the age to which he belonged [...]" (Harrison, 1996: 10).
} 
spirituale sia oggi esaurita e interamente fuori moda? Saremmo in errore se lo pensassimo; il sentimento che la vita umana, anche nella società moderna, avanzata, protetta, non sia ciò che dovrebbe essere, che ci sia qualcosa di drasticamente sbagliato nell'esistenza dell'uomo contemporaneo, è ancora assai vivo, ed anzi si accresce con il costante perfezionamento della società totalmente organizzata. In quel senso il messaggio di Michelstaedter, per quanto radicato in un insieme di circostanze culturali assai specifiche, non è affatto in procinto di perdere la sua forza, anzi ne guadagna costantemente.

Ma c'è anche dell'altro. C'è la parte meta-storica del pensiero di Michelstaedter, che non si esaurisce nella rivolta contro l'esistente, e che (per quanto sia venuta a galla qua e là nello sviluppo storico della filosofia, e soprattutto a partire dalla seconda metà del diciannovesimo secolo e per tutto il corso del ventesimo) non può essere compressa entro i limiti di nessuna teoria specifica, di nessuna scuola o posizione filosofica, ma che tuttavia esprime una funzione essenziale del discorso filosofico: è il pensiero al limite, il pensiero dell'impensato e dell'impensabile; il tentativo anticartesiano di pensare al di là di ciò che sembra giusto e ovvio, di ciò che naturalmente appare vero e certo $^{21}$.

Si disegnano quindi due "funzioni" del pensiero michelstaedteriano: una storico-antropologica (disagio della civiltà e rifiuto della modernità) e una teorico-gnoseologica (esperienza del limite e bisogno dell'oltre). Se la prima non è ancora superata, nel senso che la società contemporanea non ha trovato risposta adeguata all'anelito verso un'esitenza più autentica, la seconda è verosimilmente connaturata allo sforzo filosofico stesso, nella sua più pura determinazione: in altri termini, finché ci sarà pensiero non esclusivamente strumentale, ci sarà

21 "Ma cogito non vuol dire "So"; cogito vuol dire cerco di sapere: cioè manco del sapere: non so." (Michelstaedter, 1982: 102). 
anche la tensione a pensare l'impensabile, l'impossibile, l'inaccettabile. ${ }^{22}$

Ora, la prima funzione (apparentemente quella dominante nel discorso di Michelstaedter, ed anche quella a cui è stato dato maggior risalto dalla critica) non basta, a mio avviso, a giustificare il perdurante interesse nei confronti del goriziano. Non intendo certo mettere in dubbio la sincerità della sua passione anti-conformista; ma altri (e, fra questi, Rousseau, Nietzsche, Weininger, per citare solo alcuni nomi) hanno indubbiamente saputo esprimere con ben maggiore coerenza e capacità di convincere il dubbio radicale circa la morale, la pedagogia e in generale il comportamento sociale considerati normali. Michelsteadter aggiunge naturalmente alcuni spunti originali, ma sostanzialmente non perviene a coagulare il proprio pensiero in modi altrettanto persuasivi. Ora, paradossalmente, proprio questa incapacità di persuadere è la contropartita indispensabile, il rovescio della medaglia, della persuasione vera, così come - vorrei suggerire - lui la intende. Ed è per questo che La persuasione e la rettorica, libro pieno di bellezza, esattezza e poesia, ma anche ispido, ostico e logicamente insoddisfacente, è esattamente come dev'essere: perfetto nella propria penosa incompiutezza.

22 Mi permetto di far qui riferimento al mio Heidegger and Derrida on Philosophy and Metaphor: Imperfect Thought (Stellardi, 2000), dove il letore troverà una discussione dettagliata della natura e dello statuto del discorso filosofico. 
La cosa si spiega se si riesce a percepire, sotto lo strato superficiale della ribellione (adolescenziale?) contro l'ordine stabilito e le idee ricevute, una corrente più profonda (corrispondente alla seconda "funzione" sopra menzionata) di "pensiero del limite". Il pensiero del limite deve, per sua natura, in parte almeno sfuggire all'assise ideologica che Jacques Derrida ha definito logocentrica ${ }^{23}$. Questo è possible soltanto se si riesce a pervertire - ma solo fino a un certo punto - l'operazione della ragione, e in particolare dei suo strumenti primari: il linguaggio, la sintassi, la grammatica, l'uso.

Si veda Derrida, 1967a, 1967b, 1972. 
Il pensiero del/al limite si sforza di sfuggire alla forza centripeta del sistema in vari modi: in Heidegger, mediante la prossimità al dire poetico; in Derrida, mediante l'orizzonte dell' "indecidibile"; e in Michelstaedter, mediante la manipolazione dell'ordine discorsivo logico-sintattico-retorico. La difficoltà linguistica e stilistica della Persuasione è, quindi, non tanto tollerabile (in effetti, può darsi benissimo che essa risulti del tutto intollerabile a un certo numero di lettori), ma invece necessaria, così come lo è la relativa oscurità di molte sue articolazioni ${ }^{24}$. Il procedere a volte assurdamente convoluto, a volte volutamente pedestre e quasi infantile, ha l'effetto di sottrarre il testo all'orizzonte di attesa predeterminata del lettore veramente ricettivo: e così si aprono possibilità di risonanza non completamente dominate e risolte dall'istanza di controllo logico. In altri termini, sentiamo che il pensiero di Michelstaedter si avvicina asintoticamente ${ }^{25}$

24 Con questo, naturalmente, non intendo negare la validità di uno studio delle specifiche coordinate linguistiche all'interno delle quali si sviluppa il pensiero di Michelstaedter. Si veda, ad esempio, il lavoro di Laura Furlan (Furlan, 1999: 250 sgg.): "La lingua di Michelstaedter [...] può essere definita come il risultato dell'interazione continua tra le due forme di plurilinguismo, che il giovane vive e sperimenta (ibidem, 250).

25 L'idea che la riflessione di Michelstaedter esiga un cammino infinito non è nuova; si veda, a titolo d'esempio recente, Piero Pieri: "Il persuaso rappresenta una idea-limite dell'uomo come forma e significato globale dell'essere" (Pieri, 1989: 133; anche Giuseppe Pulina parla di "situazione-limite": Pulina, 1996: 22). II mio approccio mette l'accento non tanto sulla difficoltà di realizzare un arduo ideale umano, quanto sulla paradossale impossibilità di realizzarlo senza tradirlo allo stesso tempo: in altri termini, forse la persuasione (e l'autentico pensiero filosofico, che in questo senso finiscono per coincidere) non consistono affatto nel raggiungere ad ogni costo uno stato finale, ma invece nell'eroica accettazione del dovere di mantenersi costantemente in cammino; 0 , in altri termini, nel doppio sforzo perenne di avvicinare quell'ideale, e di NON realizzarlo in nessuna forma definitiva. Questa interpretazione sembra contraddetta dalla retorica michelstaedteriana del "permanere" e del "consistere", dal valore assoluto attribuito al "presente" (vedi nota 17); ma si tratta forse di un malinteso. Nell'evidente impossibilità di realizzare l'ideale assoluto, il cammino infinito è, forse, l'unico modo di permanere e di consistere in un presente che non sia totalmente incompatibile con la "persuasione": purché peró non sia nullificato in partenza dalla dipendenza strumentale da un fine utilitario. II che conferma che all'ideale della persuasione non può corrispondere, per Michelstaedter, un concreto progetto politico o sociale, e neppure individuale. L'"imperfezione" della proposta del goriziano è dunque non un difetto, ma una 
al punto in cui, simultaneamente, finirebbe per perdere ogni punto d'appoggio razionale, e per trovare quella verità che così faticosamente va cercando. Perché questa è la caratteristica della ricerca vera: che non si sa quale sarà il punto d'arrivo. Ma quel punto, naturalmente, non sarà mai raggiunto in questo caso, né potrebbe esserlo senza vanificare la ragione profonda della ricerca stessa. La (faticosa) liberazione dalle briglie del già noto e dell'apparentemente indiscutibile non può realizzarsi mediante una semplice sostituzione di un noto con un altro noto, di un sistema con un altro sistema, di un'ideologia con un'altra ideologia. La libertà è il dolore del cammino infinito; altrimenti, si tratta semplicemente di un'altra schiavitù.

$\mathrm{Ma}$ anche questa formulazione è inesatta. In realtà, non si può scegliere fra il sistema e il non-sistema, fra l'al di qua e l'al di là; o meglio, la scelta veramente radicale dell'al di là della ragione corrisponderebbe al silenzio, o al non senso; alla morte. Onde il paradosso, il double bind: il perseguimento di questo particolare pensiero (il pensiero al limite) richiede l'uso della ragione, ma allo stesso tempo anche un consapevole e misurato tradimento di essa. Bisogna andare al di là del limite, restando contemporaneamente al di quà; nessuna soluzione potrà essere soddisfacente.

Gli aspetti sconcertanti del testo fanno parte del percorso di esplorazione intrapreso da Michelstaedter, come anche dell'esperienza di lettura di chi ne ritenti l'impervio cammino, e ne costituiscono un aspetto inalienabile. Per questa ragione, elucidare, spiegare, chiarire il pensiero di Michelstaedter, per quanto necessario, in un certo modo è fargli torto: è riportarlo (strappandolo al regno dell'intuizione e della verità non rivelata) nell'alveo dela logica aristotelica, del buon senso, della grammatica, da cui con ogni fibra del suo essere vuole sfuggire. Ma, d'altra parte, si tratta di un torto che, in una certa misura, non possiamo risparmiare a Michelstaedter, o finiremmo per scadere in un

caratteristica essenziale del suo pensiero. 
facile misticismo dell'indicibile. Alla ragione, in sede di analisi critica, non si può rinunciare completamente.

E dunque non si può evitare un'ultima domanda, la più spinosa: nel chiaroscuro del pensiero al limite, come decidere se la strada intrapresa da Michelstaedter sia quella giusta? Quali mezzi (non arbitrari, non illusori) abbiamo a disposizione, se la ragione stessa appare più come una catena da rifiutare, che un sostegno fidato? In fin dei conti, abbracciare una posizione "al limite" (come quella michelstaedteriana) può solo essere una scelta del tipo della "non scelta", nel senso che non ci può essere prova razionale definitiva che la scelta sia corretta, che abbiamo ragione di compierla. Dunque, se la abbracciamo, si tratta di un atto simile a quello di tipo kantiano: come nel caso di un imperativo categorico, siamo di fronte a qualcosa che si impone alla nostra ragione, al di là di ogni possibilità di giustificarlo razionalmente.

$\mathrm{Si}$ badi che Michelstaedter non è preparato a rinunciare a ogni certezza e a suggerirci la soluzione del cinismo o del disimpegno: propone invece un rifiuto delle certezze comuni, ma in favore del riconoscimento (irrazionale) del valore assoluto di altre (ma ingiustificabili) certezze. Impedendoci dunque di riposare in una posizione tanto di cieco conformismo, quanto di facile anticonformismo, non ci consente però neppure di rifugiarci in una dimensione di agnosticismo intellettuale e etico: ci obbliga a stare perennemente sul limite, ed è la posizione più scomoda di tutte.

Ecco perché La persuasione è esattamente come dovrebbe essere: un libro bello a tratti, ma duro, ostinato, ostile, sbagliato, inaccettabile, incompleto. È esattamente coì com'è, con tutte le sue difficoltà e domande senza risposta, che ci sfida, non tanto a accogliere o rifiutare una certa specifica proposta filosofica o politica, ma piuttosto a cercare di pensare oltre ciò che così chiaramente è per noi lo schema corretto, accettabile, sicuro: a pensare oltre la sicurezza e la comodità, oltre la morale e l'equità correnti, per cercare di raggiungere forse (ma quando? ma come?) un regno dove la vita, l'amore e la giustizia 
acquistino un significato diverso e più intenso. O dove, forse, tutto è perduto, inclusa la vita.

$\mathrm{Ne}$ vale la pena? Non ci è veramente richiesto di seguire fino in fondo Michelstaedter nel suo solitario cammino. Ma, dalla sicurezza del nostro tavolino, possiamo contemplare il suo eroico sforzo e la sua tragica discesa, e per un momento almeno sapere che essere uomini significa anche non accettare ciecamente il sentiero che ci è stato assegnato.

Non possiamo nasconderci il grave pericolo associato a una posizione, come quella di Michelstaedter, di radicale rifiuto della "normalità" e del "buon senso". Al salto nel vuoto fuori dai binari prefissati dal "consorzio civile" (che Michelstaedter chiama, senza mezzi termini, la "comunella dei malvagi") - e sia pure con le migliori intenzioni - possono seguire le peggiori conseguenze: il fascismo, il comunismo sovietico, Pol Pot, tutti i mostri dell'età presente, e tutti quelli che per ora solo possiamo immaginare e temere, e che abbiamo il dovere di evitare.

Come proposta concreta, quella di Michelstaedter è, oltre che poco perspicua nei suoi contorni concreti, anche inevitabilmente fallimentare; ma, da un lato, l'insopprimibile anelito di vita vera che la sottende è qualcosa di cui l'uomo moderno ha sempre (e forse sempre più) bisogno; e, dall'altro, l'eroica determinazione a sfuggire alle categorie prestabilite è forse la più pura incarnazione della pulsione filosofica, e dunque del pensiero umano.

A Michelstaedter non sfugge né la tragica insuperabilità del double bind in cui si trova, né l'ironica impotenza della propria condizione di rivoltato cronico. $\mathrm{Sa}$, in effetti, che la persuasione a cui anela non può derivare logicamente dallo sforzo di ricerca da lui condotto: "[...] e nel migliore dei casi avrò fatto... una tesi di laurea." (Michelstaedter, 1982: 36). Ma è proprio perché questa non è una "buona, normale o brillante tesi di laurea", che il libro ha ancora qualcosa da dirci a proposito tanto della nostra condizione di individui sociali, quanto del nostro scomodo destino di esseri pensanti. 
Perché, dunque, a ventunesimo secolo ormai inoltrato, ancora leggere Michelstaedter? Perché non abbiamo ancora finito (né potremo finire mai) di interrogare il nostro rapporto con l'ambiente naturale e sociale (la distinzione fra $\mathrm{i}$ due sta precisamente al cuore dell'interrogativo) in cui viviamo; e anche (soprattutto?) perché pochi altri libri ci costringeranno altrettanto inesorabilmente a far fronte alla più grande fatica, dopo quella di vivere: quella di pensare.

(Oxford University)

\section{Bibliografia}

AA.VV.

Bertinotti, Fausto

e Gianni, Alfonso

Bini, Daniela

Brianese, Giorgio

Campailla, Sergio
1994. Sotto il segno di Michelstaedter. Il valore di una identità, a cura di Toni Iermano. Cosenza: Edizioni Periferia.

1996. Carlo Michelstaedter. Pordenone: Studio Tesi.

2002. Per una pace infinita. Firenze: Ponte alle Grazie.

1992. Carlo Michelstaedter and the failure of language. Gainesville: University Press of Florida.

1985. L'arco e il destino: interpretazione di Michelstaedter. Abano Terme, Padova: Francisci.

1973 Pensiero e poesia di Carlo Michelstaedter. Bologna : Pàtron. 


\begin{tabular}{|c|c|c|}
\hline Campailla, Sergio & 1981. & $\begin{array}{l}\text { A ferri corti con la vita. Gorizia: Il } \\
\text { Comune. }\end{array}$ \\
\hline Cerruti, Marco & 1987. & $\begin{array}{l}\text { Carlo } \quad \text { Michelstaedter. } \\
\text { Mursia. }\end{array}$ \\
\hline Derrida, Jacques & 1967a. & $\begin{array}{l}\text { De la grammatologie. Paris: Editions } \\
\text { de Minuit. }\end{array}$ \\
\hline Derrida, Jacques & 1967b. & $\begin{array}{l}\text { L'écriture et la différence. Paris: } \\
\text { Editions du Seuil. }\end{array}$ \\
\hline Derrida, Jacques & 1972. & $\begin{array}{l}\text { Marges, de la philosophie. Editions } \\
\text { de Minuit. }\end{array}$ \\
\hline Furlan, Laura & 1999. & $\begin{array}{l}\text { Carlo Michelstaedter. L'essere } \\
\text { straniero di un intellettuale moderno. } \\
\text { Trieste: LINT. }\end{array}$ \\
\hline Innella, Francesco & 1995. & $\begin{array}{l}\text { Michelstaedter: frammenti di una } \\
\text { filosofia oscura. Salerno, Roma: } \\
\text { Edizioni Ripostes. }\end{array}$ \\
\hline Michelstaedter, Carlo & 1958. & $\begin{array}{l}\text { Opere, a cura di Gaetano Chiavacci. } \\
\text { Firenze: Sansoni }\end{array}$ \\
\hline Michelstaedter, Carlo & 1982. & $\begin{array}{l}\text { La persuasione e la rettorica, a cura } \\
\text { di Sergio Campailla. Milano: Adelphi. }\end{array}$ \\
\hline Michelstaedter, Carlo & 2004. & $\begin{array}{l}\text { Persuasion and Rhetoric, transl. by } \\
\text { Russell Scott Valentino, Cinzia } \\
\text { Sartini Blum and David J. Depew. } \\
\text { New Haven: Yale University Press. }\end{array}$ \\
\hline
\end{tabular}


Eco, Umberto

Eco, Umberto

Harrison, Thomas

Michelis, Angela

Muzzioli, Francesco

Negri, Antimo

Papini, Giovanni

Pieri, Piero

Pirandello, Luigi

Pulina, Giuseppe
1985. Come si fa una tesi di laurea. Milano: Bompiani.

1990. I limiti dell'interpretazione. Milano: Bompiani.

1996. 1910: The Emancipation of Dissonance. Berkeley: University of California Press.

1997. Carlo Michelstaedter. Il coraggio dell'impossibile. Roma: Città Nuova Editrice.

1987. Michelstaedter. Lecce : Milella.

1996. Il lavoro e la città. Un saggio su Carlo Michelstaedter. Roma, Lavoro.

1910. "Un suicidio metafisico", in Il Resto del Carlino, 5 novembre 1910.

1989. La scienza del tragico. Saggio su Carlo Michelstaedter. Bologna: Cappelli.

1989. Quando si è qualcuno, La favola del fliglio cambiato, I giganti della montagna. Milano: Oscar Mondadori.

1996. L'imperfetto pessimista. Saggio sul pemsiero di Carlo Michelstaedter. Poggibonsi: Lalli editore. 
Raschini, M. Adelaide 2000. Michelstaedter. Firenze: Marsilio.

Semeraro, Licia

1986. Lo svuotamento del futuro. Note su Michelstaedter. Lecce: Milella.

Stella,Vittorio

2002. Carlo Michelstaedter. Milano: FERV.

Stellardi, Giuseppe

2000. Heidegger and Derrida on Philosophy and Metaphor: Imperfect Thought. Amherst, New York: Humanity Books.

Taviani, Giovanna

2002. Michelstaedter. Palermo: Palumbo.

Tortora, Giuseppe

1994. Kierkegaard and Schopenhauer on Hegelianism, in Metalogicon, VII, 1: 69-84 (ora disponibile anche su internet:

http://www.filosofia.unina.it/ tortora/saggi/kierkegaard.html) 